\title{
Photoacoustic microscopy with enhanced resolution and imaging depth aided by optical clearing
}

Yong Zhou, Junjie Yao, Lihong V. Wang

Yong Zhou, Junjie Yao, Lihong V. Wang, "Photoacoustic microscopy with enhanced resolution and imaging depth aided by optical clearing," Proc. SPIE 8943, Photons Plus Ultrasound: Imaging and Sensing 2014, 89433A (3 March 2014); doi: $10.1117 / 12.2036636$

SPIE. Event: SPIE BiOS, 2014, San Francisco, California, United States 


\title{
Photoacoustic Microscopy with Enhanced Resolution and Imaging Depth Aided by Optical Clearing
}

\author{
Yong Zhou, Junjie Yao, Lihong V. Wang* \\ Optical Imaging Laboratory, Department of Biomedical Engineering, Washington University in \\ St. Louis, One Brookings Drive, St. Louis, MO 63130, USA \\ *Address all correspondence to: lhwang@wustl.edu
}

\begin{abstract}
Both the spatial resolution and maximum penetration depth of optical-resolution photoacoustic microscopy (ORPAM) deteriorate sharply with depth due to strong light scattering in tissue. To reduce tissue scattering, we propose to use glycerol as an optical clearing agent in OR-PAM. Our results show that the imaging performance of OR-PAM can be greatly enhanced by optical clearing both in vitro and in vivo.
\end{abstract}

Keywords: optical-resolution photoacoustic microscopy, penetration depth, lateral resolution, optical clearing

\section{Introduction}

Photoacoustic microscopy (PAM) is a newly developed imaging modality that can provide structural, ${ }^{1-3}$ molecular, ${ }^{4}$ functional, ${ }^{5-8}$ and metabolic information. ${ }^{9}$ Based on its focusing mechanism, PAM can be divided into two different implementations: acoustic-resolution PAM (AR-PAM) and optical-resolution PAM (OR-PAM). In OR-PAM, the lateral resolution is determined by the diffraction-limited optical focusing. As photons travel in tissue, the focusing capability degrades due to optical scattering. In addition, the maximum penetration depth of OR-PAM in biological tissue is mainly limited by the optical transport mean free path, affected by both absorption and scattering. Since the tissue scattering is typically an order of magnitude larger than the absorption, it is the scattering that predominantly limits the penetration depth of OR-PAM.

To reduce the scattering, tissue optical clearing (TOC) techniques have been widely used in many high-resolution optical imaging modalities, such as laser speckle contrast imaging and optical coherence tomography. ${ }^{10}$ The basic mechanism of TOC is to diffuse a high-refractive-index optical clearing agent (OCA) into the tissue, which reduces the refractive index mismatch between intracellular components and extracellular fluids, and thus decreases the scattering. In addition, tissue dehydration caused by OCAs has also been used to illustrate the mechanism of TOC. In this paper, for the first time, we demonstrate that both the imaging depth and lateral resolution of OR-PAM can be enhanced by using a glycerol-water solution $(88 \%$, vol/vol), which is a commonly used OCA in TOC.

\section{System}

We implemented this idea on a previously reported OR-PAM system (Fig. 1). Briefly, a tunable dye laser (CBR-D, Sirah, GmbH.) pumped by a Nd:YLF laser (INNOSAB, Edgewave, GmbH.) was the light source. After being focused by a condenser lens, filtered by a pinhole, and reflected by a mirror, the laser beam was finally focused by an objective lens into the sample. Ultrasonic detection was achieved by a wide-band ultrasonic transducer (V214-BC, Panametrics-NDT Inc.), which was placed confocally with the optical objective lens. Each laser pulse yielded a onedimensional depth-resolved PA image (A-line) by recording the time course of photoacoustic signals. A threedimensional (3D) image was obtained by raster scanning the sample and piecing together A-lines.

Photons Plus Ultrasound: Imaging and Sensing 2014, edited by Alexander A. Oraevsky, Lihong V. Wang, Proc. of SPIE Vol. 8943, 89433A - (C) 2014 SPIE · CCC code: 1605-7422/14/\$18 - doi: 10.1117/12.2036636 


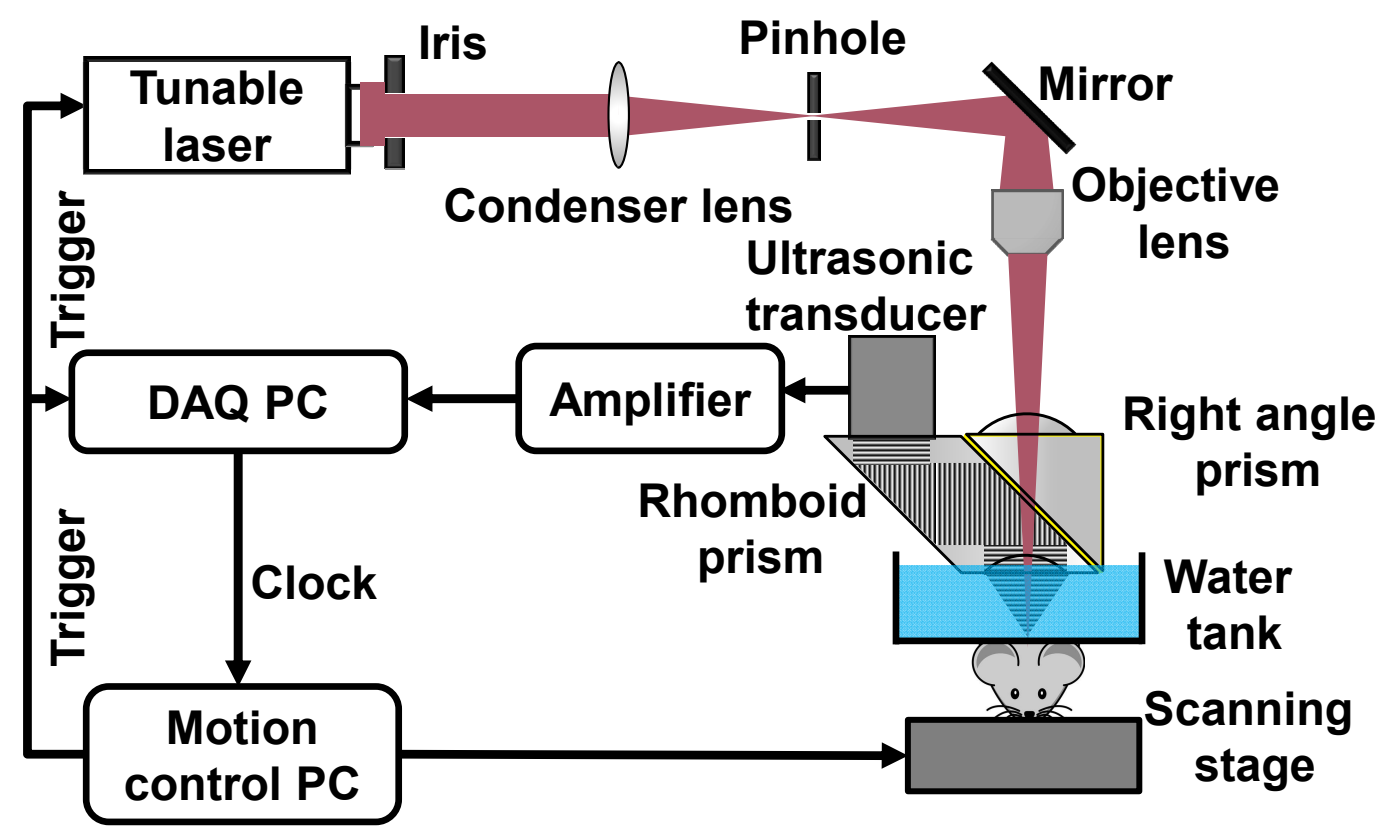

Fig. 1. System schematic used in the experiment.

\section{Phantom experiment}

First, in a phantom experiment, a United States Air Force (USAF-1951, Edmund) resolution test target covered by a piece of freshly harvested mouse skin was imaged at $570 \mathrm{~nm}$. The phantom was immersed in the glycerol-water solution. Fig. 2 shows that the average total PA signal amplitude from the United States Air Force resolution test target versus time; after $180 \mathrm{~min}$, the amplitude increased by more than two fold over the baseline value. Note that the PA signals were still increasing at the point when we stopped the experiment. This phantom experiment clearly illustrates that the scattering of the tissue was reduced by optical clearing, and thus the penetration depth was enhanced.

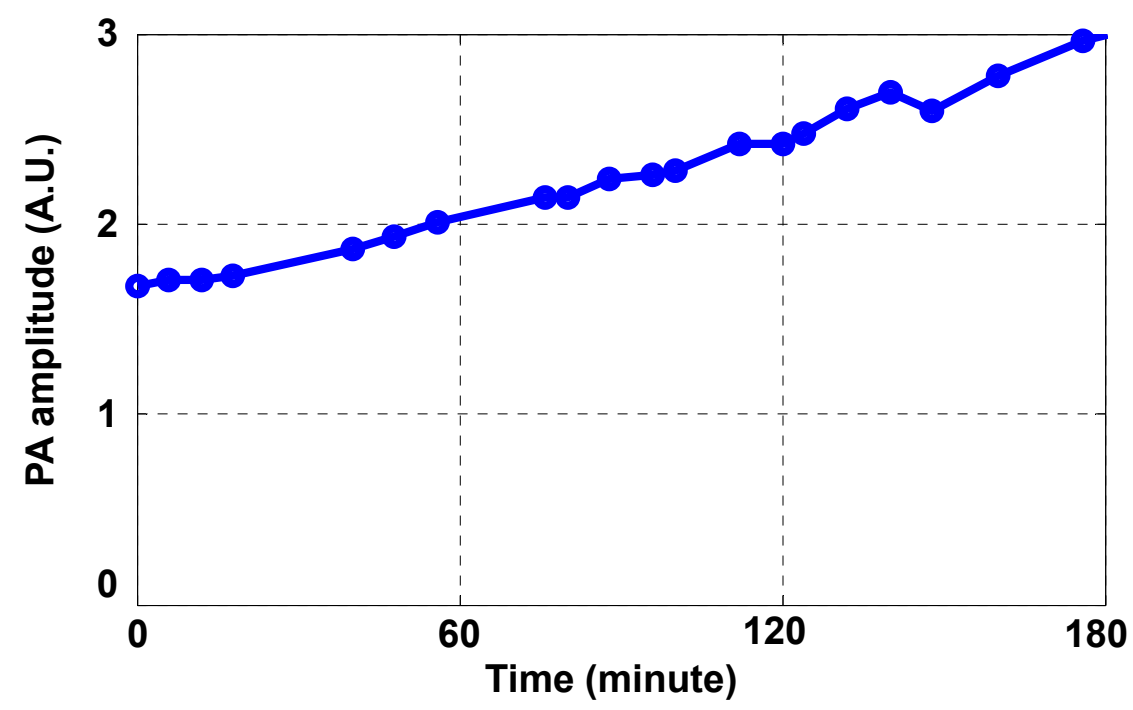

Fig. 2. Optical clearing aided PAM of a United States Air Force resolution test target covered by mouse skin. (e) Time course of the PA amplitude averaged over the whole image during optical clearing. The higher PA amplitude indicates that the optical clearing increased penetration depth. 


\section{In vivo experiment}

Next, we used a nude mouse in an in vivo experiment. All experimental animal procedures were carried out in conformance with the laboratory animal protocol approved by the Animal Studies Committee of Washington University in St. Louis. Because the diffusion of glycerol across the epidermal layer is very slow, we directly injected the glycerol-water solution into the mouse scalp to create a local optical clearing window.
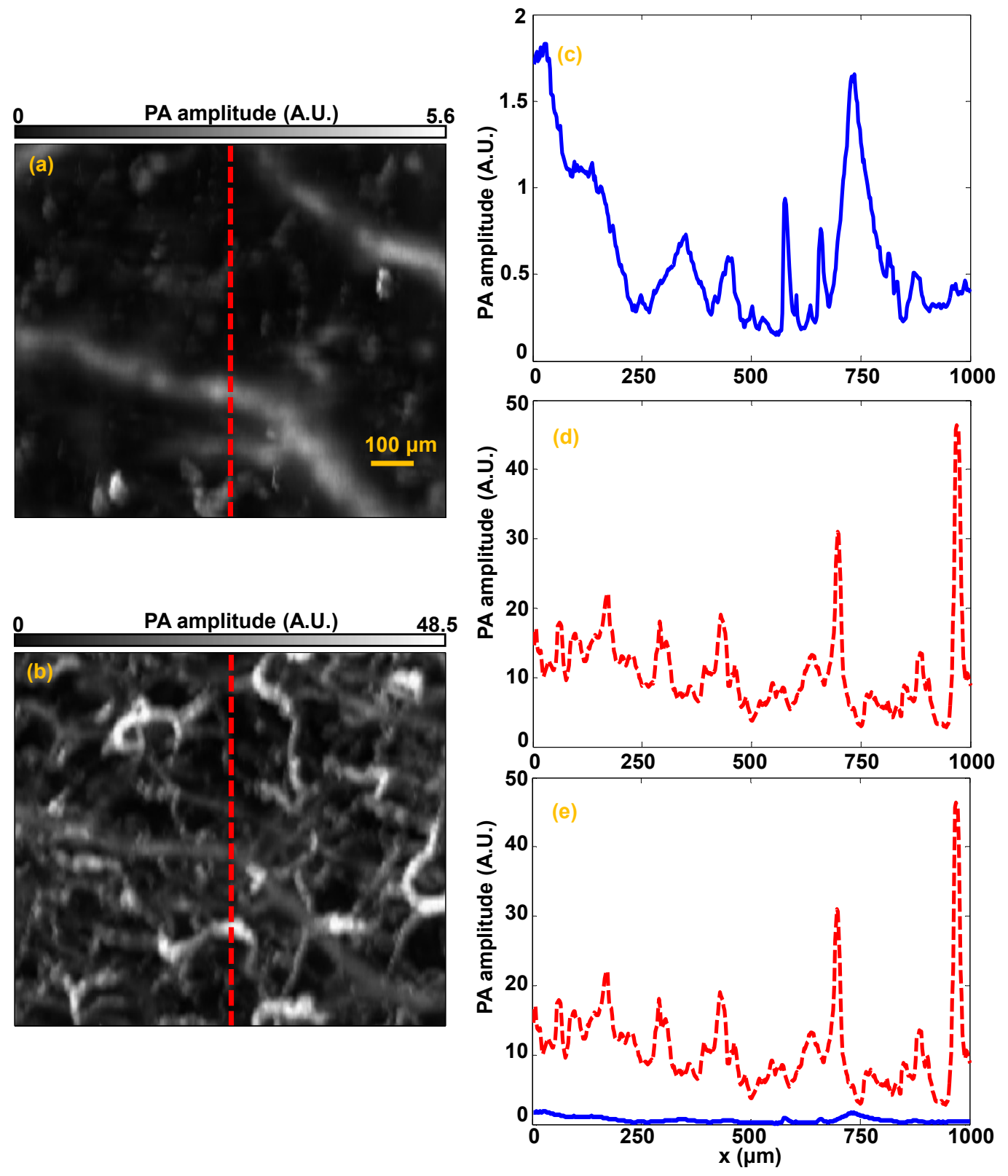

Fig. 3. Optical clearing reveals more capillaries. (a) PAM image before optical clearing. (b) PAM image with capillaries after optical clearing. (c-d) PA amplitudes along the red dashed lines in (a-b). (e) Combined curves in (c) and (d).

The enhanced PA detection sensitivity after optical clearing enabled better capillary imaging [Fig. 3]. Fig. 3(a) and (b) the maximum-amplitude-projection (MAP) of the PA images of blood vessels in the mouse scalp before and after optical clearing, respectively. The large vessels in the center of both images indicate that they are from the same area in the mouse scalp. As shown in Fig. 3(a), very few capillaries can be detected before optical clearing. 
However, after optical clearing, more capillaries can be clearly observed, as shown in Fig. 3(b). As shown in Figs. 3(c)-(e), the vessel density had increased by about 10 times after clearing and the averaged PA signal amplitude from individual capillaries had increased by a factor of 17 . The much denser capillaries and stronger PA amplitudes in Fig. 3(e) indicate that, after optical clearing, the local laser fluence at the capillaries was increased. Therefore, we show that the attenuation coefficients were reduced after optical clearing. Because optical clearing did not affect absorption cross section, we concluded that scattering cross section was reduced after optical clearing.

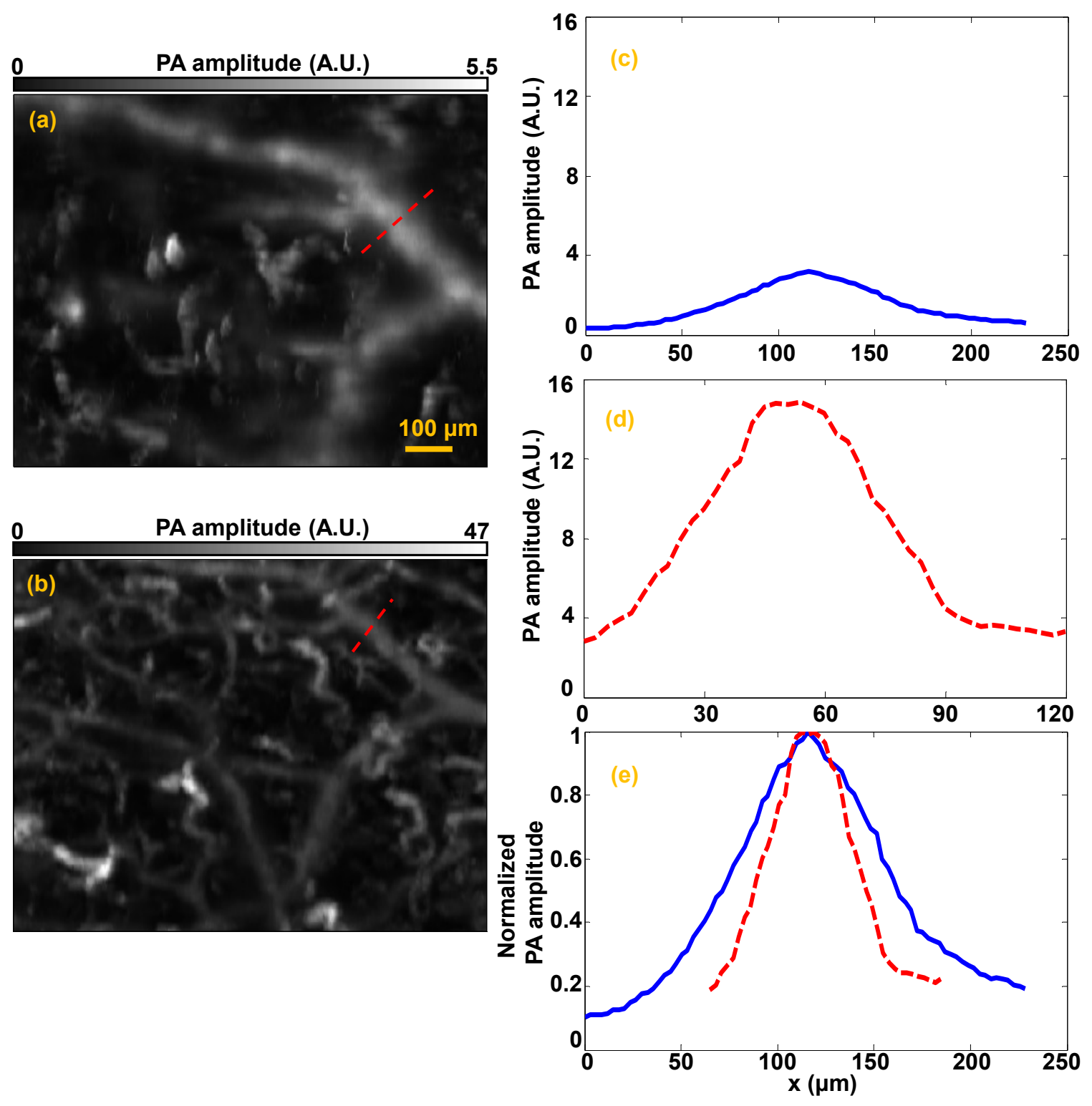

Fig. 4. Optical clearing improves the lateral resolution for deep vessels. (a) PAM image before optical clearing. (b) PAM image with parts of capillary layer digitally removed after optical clearing. (c-d) PA amplitudes along the red dashed lines in (a-b). (e) Combined curves in (c) and (d) after normalization.

We further analyzed the lateral resolution enhancement after optical clearing. Fig. 4(a) and (b) the maximumamplitude-projection (MAP) of the PA images of blood vessels in the mouse scalp before and after optical clearing, respectively. As shown in Fig. 4(a) and (b), we found that the vessels before optical clearing were much more blurred than those after optical clearing. Again, the averaged PA amplitude was increased by about 4 times [Figs. 
4(c)-(d)]. More importantly, we also estimated the lateral resolution improvement by comparing the vessel diameters. As shown in Fig. 5(e), a representative vessel had nominal diameters of $57 \mu \mathrm{m}$ and $93 \mu \mathrm{m}$ before and after clearing, respectively. We can see that measured vessel diameter decreased at least by 1.7 times after optical clearing. Because the measured vessel diameter was the convolution of the actual lateral resolution with the true vessel diameter, the actual resolution improvement should be better than 1.7 times.

There are several widely used OCAs, such as glycerol, glucose, and propylene glycol. Among these agents, glycerol has both good clearing efficiency and a long effective time. In addition, because glycerol has also been widely used in the medical, pharmaceutical, and chemical fields, it can be easily accessed. Thus, in all experiments, we elected to use glycerol as the OCA to reduce tissue scattering. To achieve a relatively good clearing result and to reduce the potential tissue damage caused by dehydration, a diluted glycerol-water solution $(88 \%$, vol/vol) was used. A higher glycerol concentration may enhance the clearing effect with a higher risk of tissue damage.

Because glycerol penetrates very slowly through epidermal tissue, we directly injected the glycerol solution into the tissue to accelerate optical clearing. However, injection may lead to side effects, such as bleeding and edema. To use glycerol without injection, we can explore either physical or chemical approaches, such as using a micro needle array or a DMSO admixture, respectively. In addition to glycerol, next we will try other optical clearing media, such as glucose solution.

Our results show that both the penetration depth and lateral resolution of PA imaging can be enhanced by TOC. For back-scattering based optical imaging modalities such as optical coherence tomography, optical clearing can provide deeper penetration depth as well. However, it may destroy some of the intrinsic scattering contrasts that these modalities rely on, which can result in potential information loss. By contrast, photoacoustic imaging is based on optical absorption with $100 \%$ sensitivity. Since the optical absorption is largely kept intact in the optical clearing procedure, information loss is avoided. Not only can PA imaging benefit from optical clearing, it can also be used to study the physiological effects induced by optical clearing. With enhanced penetration depth and resolution, PAM will become more valuable for structural, molecular, functional, and metabolic imaging.

\section{Acknowledgements}

The authors would like to thank Prof. James Ballard for manuscript editing. We also appreciate technical assistance and useful discussions from Jinyang Liang and Chi Zhang. This research was supported by the National Institutes of Health Grants DP1 EB016986 (NIH Director's Pioneer Award), R01 EB008085, R01 CA134539, U54 CA136398, and R01 CA159959. L.V.W. has a financial interest in Microphotoacoustics, Inc. and Endra, Inc., which, however, did not support this work.

\section{References}

[1]. L. H. V. Wang and S. Hu, "Photoacoustic tomography: In vivo imaging from organelles to organs," Science 335(6075), 1458-1462 (2012)

[2]. Y. Zhou, J. J. Yao and L. H. V. Wang, "Optical clearing-aided photoacoustic microscopy with enhanced resolution and imaging depth," Opt Lett 38(14), 2592-2595 (2013)

[3]. C. Zhang, Y. Zhou, C. Y. Li and L. H. V. Wang, "Slow-sound photoacoustic microscopy," Appl Phys Lett 102(16), (2013)

[4]. Y. Zhou, C. Zhang, D. K. Yao and L. H. V. Wang, "Photoacoustic microscopy of bilirubin in tissue phantoms," J Biomed Opt 17(12), (2012)

[5]. J. Y. Liang, Y. Zhou, A. W. Winkler, L. D. Wang, K. I. Maslov, C. Y. Li and L. H. V. Wang, "Randomaccess optical-resolution photoacoustic microscopy using a digital micromirror device," Opt Lett 38(15), 2683-2686 (2013)

[6]. Y. Zhou, J. Y. Liang, K. I. Maslov and L. H. V. Wang, "Calibration-free in vivo transverse blood flowmetry based on cross correlation of slow time profiles from photoacoustic microscopy," Opt Lett 38(19), 3882-3885 (2013) 
[7]. J. Y. Liang, Y. Zhou, K. I. Maslov and L. H. V. Wang, "Cross-correlation-based transverse flow measurements using optical resolution photoacoustic microscopy with a digital micromirror device," $J$ Biomed Opt 18(9), (2013)

[8]. J. J. Yao, H. X. Ke, S. Tai, Y. Zhou and L. H. V. Wang, "Absolute photoacoustic thermometry in deep tissue," Opt Lett 38(24), 5228-5231 (2013)

[9]. J. J. Yao, J. Xia, K. I. Maslov, M. Nasiriavanaki, V. Tsytsarev, A. V. Demchenko and L. V. Wang, "Noninvasive photoacoustic computed tomography of mouse brain metabolism in vivo," Neuroimage 64(257-266 (2013)

[10]. D. Zhu, K. V. Larin, Q. M. Luo and V. V. Tuchin, "Recent progress in tissue optical clearing," Laser Photonics Rev 7(5), 732-757 (2013) 